\title{
Solving Linear Fredholm-Stieltjes Integral Equations of the Second Kind by Using the Generalized Midpoint Rule
}

\author{
Avyt Asanov, M.Musa Abdujabbarov ${ }^{*}$ \\ International Atatuk - Alatoo University
}

Received: April 20, 2015 / Accepted: May 18, 2015 / Published: November 25, 2015.

\begin{abstract}
In this paper, the approximate solution to the linear fredholm-stieltjes integral equations of the second kind (LFSIESK) by using the generalized midpoint rule (GMR) is introduced. A comparison results depending on the number of subintervals " $n$ " are calculated by using Maple 18 and presented. These results are demonstrated graphically in a particular numerical example. An algorithm of this application is given by using Maple 18.
\end{abstract}

Keywords: Approximate solutions, linear fredholm-stieltjes integral equations, midpoint rule.

\section{Introduction}

An integral equations theory is one of the most active research fields which is based on analysis, functions theory and functional analysis [1]. Moreover, since the partial differential equations can be transformed into integral equations, this also makes integral equations more interesting [1]. Sometimes it may be difficult to solve integral equations analytically, so approximation methods, which has expanded very rapidly during this computer era [2], are used to solve the integral equations. There are many approximation methods to solve linear Fredholm integral equations of the second kind: projection, Nyström [3], Midpoint [4] methods etc. However, there are very few of them that solve LFSIESK: generalized trapezoid rule [5], [6].

\section{Solving Lfsiesk by Using the GMR}

\subsection{Given Lfsiesk}

$$
\begin{gathered}
u(x)=\int_{a}^{b} K(x, y) u(y) d g(y)+f(x), \\
x \in[a, b]
\end{gathered}
$$

Corresponding author: Muhammad Musa Abdujabbarov, International Atatuk - Alatoo University. Research field. Integral equations, scientific computing. E-mail: abdujabbarov@yahoo.com. where $K(x, y) \in C[a, b], g(y)$ is the continuous function on the closed interval $[a, b]$ which can be written as a difference of two strictly increasing functions $\varphi(y), \psi(y)$ on the closed interval $[a, b]$, $f(x)$ given function and $u(x)$ is the unknown function to be determined. Now, instead of $g(y)$ in (1), if the difference $\varphi(y)-\psi(y)$ is substituted, then it becomes of the form

$$
\begin{aligned}
& u(x)=\int_{a}^{b} K(x, y) u(y) d g(y)+f(x)= \\
& \underbrace{\int_{a}^{b} K(x, y) u(y) d \varphi(y)}_{I}-\underbrace{\int_{a}^{b} K(x, y) u(y) d \psi(y)}_{I I}+f(x)
\end{aligned}
$$

Here the integrals in (2) can be calculated separately by using the GMR [7],

$$
\begin{aligned}
& I=\int_{a}^{b} K(x, y) u(y) d \varphi(y) \approx \\
& \sum_{i=1}^{n} K\left(x, x_{2 i-1}^{*}\right) u\left(x_{2 i-1}^{*}\right)\left[\varphi\left(x_{2 i}\right)-\varphi\left(x_{2 i-2}\right)\right]
\end{aligned}
$$

where

$$
x_{2 i-1}^{*}=\varphi^{-1}\left[\frac{\varphi\left(x_{2 i}\right)+\varphi\left(x_{2 i-2}\right)}{2}\right]
$$


and

$$
\begin{aligned}
& I I=\int_{a}^{b} K(x, y) u(y) d \psi(y) \approx \\
& \sum_{i=1}^{n} K\left(x, x_{2 i-1}^{* *}\right) u\left(x_{2 i-1}^{* *}\right)\left[\psi\left(x_{2 i}\right)-\psi\left(x_{2 i-2}\right)\right]
\end{aligned}
$$

where $x_{2 i-1}^{* *}=\psi^{-1}\left[\frac{\psi\left(x_{2 i}\right)+\psi\left(x_{2 i-2}\right)}{2}\right]$

Therefore, the integral equation (2) becomes

$$
\bar{u}(x)=\sum_{i=1}^{n}\left(\begin{array}{l}
K\left(x, x_{2 i-1}^{*}\right) \bar{u}\left(x_{2 i-1}^{*}\right) \times \\
{\left[\left(x_{2 i}\right)-\varphi\left(x_{2 i-2}\right)\right]-} \\
K\left(x, x_{2 i-1}^{* *}\right) \bar{u}\left(x_{2 i-1}^{* *}\right) \times \\
{\left[\psi\left(x_{2 i}\right)-\psi\left(x_{2 i-2}\right)\right]}
\end{array}\right)+f(x)
$$

where $\bar{u}(x)$ is the approximate solution of (2).

Now, in (3), if we use the following substitution:

$$
\begin{aligned}
& A_{i}(x)=K\left(x, x_{2 i-1}^{*}\right)\left[\varphi\left(x_{2 i}\right)-\varphi\left(x_{2 i-2}\right)\right], \\
& B_{i}(x)=-K\left(x, x_{2 i-1}^{* *}\right)\left[\psi\left(x_{2 i}\right)-\psi\left(x_{2 i-2}\right)\right],
\end{aligned}
$$

for $i=1,2,3, \ldots, n$,

then the equation (3) can be written as

$$
\bar{u}(x)=\sum_{i=1}^{n}\left(\begin{array}{l}
A_{i}(x) \bar{u}\left(x_{2 i-1}^{*}\right)+ \\
B_{i}(x) \bar{u}\left(x_{2 i-1}^{* *}\right)
\end{array}\right)+f(x)
$$

If $x_{2 i-1}^{*}$ and $x_{2 i-1}^{* *}$ for $i=1,2,3, \ldots, n$ are substituted into (4), then we get a following system of linear equations

$$
\begin{gathered}
\bar{u}\left(x_{2 j-1}^{*}\right)=\sum_{i=1}^{n}\left(\begin{array}{c}
A_{i}\left(x_{2 j-1}^{*}\right) \bar{u}\left(x_{2 i-1}^{*}\right)+ \\
B_{i}\left(x_{2 j-1}^{*}\right) \bar{u}\left(x_{2 i-1}^{* *}\right)
\end{array}\right)+f\left(x_{2 j-1}^{*}\right) \\
\bar{u}\left(x_{2 j-1}^{* *}\right)=\sum_{i=1}^{n}\left(\begin{array}{c}
A_{i}\left(x_{2 j-1}^{* *}\right) \bar{u}\left(x_{2 i-1}^{*}\right)+ \\
B_{i}\left(x_{2 j-1}^{* *}\right) \bar{u}\left(x_{2 i-1}^{* *}\right)
\end{array}\right)+f\left(x_{2 j-1}^{* *}\right) \\
\text { For } j=1,2, \ldots, n
\end{gathered}
$$

If the system of linear equations (5) is converted into matrix form, then

$$
\begin{aligned}
& (I-\underbrace{\left(\begin{array}{ccc}
A_{i}\left(x_{2 j-1}^{*}\right) & \cdots & B_{i}\left(x_{2 j-1}^{*}\right) \\
\vdots & \ddots & \vdots \\
A_{i}\left(x_{2 j-1}^{* *}\right) & \cdots & B_{i}\left(x_{2 j-1}^{* *}\right)
\end{array}\right)}_{A}) \cdot \underbrace{\left(\begin{array}{c}
\bar{u}\left(x_{2 j-1}^{*}\right) \\
\vdots \\
\bar{u}\left(x_{2 j-1}^{* *}\right)
\end{array}\right)}_{U} \\
& =\underbrace{\left(\begin{array}{c}
f\left(x_{2 j-1}^{*}\right) \\
\vdots \\
f\left(x_{2 j-1}^{* *}\right)
\end{array}\right)}_{F}
\end{aligned}
$$

$$
\text { for } i, j=1,2, \ldots, n
$$

Now, the system (6) $(I-A) \cdot U=F$ has a unique solution $U=(I-A)^{-1} \cdot F$ if and only if $\operatorname{det}(I-A) \neq 0$.

Now, let $\varphi(x) \in C^{\alpha}[a, b], \psi(x) \in C^{\beta}[a, b]$ where $0<\alpha \leq 1,0<\beta \leq 1$.

Then in the

$$
A_{i}(x)=K\left(x, x_{2 i-1}^{*}\right)\left[\varphi\left(x_{2 i}\right)-\varphi\left(x_{2 i-2}\right)\right] \quad \text { and }
$$
$B_{i}(x)=K\left(x, x_{2 i-1}^{* *}\right)\left[\psi\left(x_{2 i}\right)-\psi\left(x_{2 i-2}\right)\right]$, the terms

$$
\left[\varphi\left(x_{2 i}\right)-\varphi\left(x_{2 i-2}\right)\right],\left[\psi\left(x_{2 i}\right)-\psi\left(x_{2 i-2}\right)\right]
$$

approaches 0 as $x_{2 i}-x_{2 i-2}$ approaches 0 , at least as fast as $\left|x_{2 i}-x_{2 i-2}\right|^{\alpha}$ approaches 0 .

Therefore, $A_{i}(x)$ and $B_{i}(x)$ approaches 0 as the number of subintervals " $\mathrm{n}$ " increases for all $i=1,2,3, \ldots, n$. [8], [9].

So, the coefficient matrix will be of the form $A \approx\left(\begin{array}{ccc}\approx 0 & \cdots & \approx 0 \\ \vdots & \ddots & \vdots \\ \approx 0 & \cdots & \approx 0\end{array}\right)$ and we can conclude that $\operatorname{det}(I-A) \neq 0$.

Therefore, the system (6) has a unique solution, namely $U=(I-A)^{-1} \cdot F$.

Thus, if the solution of the system of linear equations (6) is substituted back into the (4), then the general solution is defined as 


$$
\begin{aligned}
& \bar{u}(x)=\sum_{i=1}^{n} U_{i} \cdot A_{i}(x)+ \\
& \sum_{i=1}^{n} U_{n+i} \cdot B_{i}(x)+f(x)
\end{aligned}
$$

Numerical example:

Let us consider the following LFSIESK

$$
\begin{gathered}
u(x)=\int_{0}^{1}\left(1+x^{2} s\right) u(s) d(\ln (1+\sqrt{s})) \\
-\frac{x^{2}}{4}-\frac{x}{6}+x \sqrt{x}
\end{gathered}
$$

here

$$
K(x, s)=1+x^{2} s, \varphi(x)=\ln (1+\sqrt{x}) \quad \psi(x)=0
$$

and

$$
f(x)=-\frac{x^{2}}{4}-\frac{x}{6}+x \sqrt{x} .
$$

Let us take $n=4$,

then

$$
h=\frac{b-a}{2 n}=\frac{1-0}{8}=0.125
$$

And

$$
x_{2 i}=a+2 i h=0.125 \cdot 2 i
$$

for

$$
i=1,2,3,4 \text {. }
$$

If it is calculated, then it can be obtained as

$$
\begin{gathered}
x_{0}=0, x_{2}=0.25, x_{4}=0.5, \\
x_{6}=0.75, x_{8}=1.0
\end{gathered}
$$

Then, if the GMR is used to integrate (8),

$$
\begin{aligned}
& I=\int_{0}^{1}\left(1+x^{2} s\right) u(s) d(\ln (1+\sqrt{s})) \approx \\
& \sum_{i=1}^{4} K\left(x, x_{2 i-1}^{*}\right) u\left(x_{2 i-1}^{*}\right)\left[\varphi\left(x_{2 i}\right)-\varphi\left(x_{2 i-2}\right)\right]
\end{aligned}
$$

where

$$
x_{2 i-1}^{*}=\varphi^{-1}\left[\frac{\varphi\left(x_{2 i}\right)+\varphi\left(x_{2 i-2}\right)}{2}\right]
$$

if calculated, then it can be obtained as

$$
\begin{array}{r}
x_{1}^{*}=0.0505, x_{3}^{*}=0.3602, \\
x_{5}^{*}=0.6159, x_{7}^{*}=0.8683
\end{array}
$$

So the equation (8) becomes

$$
\begin{aligned}
& \bar{u}(x)= \\
& \sum_{i=1}^{4} K\left(x, x_{2 i-1}^{*}\right) u\left(x_{2 i-1}^{*}\right)\left[\varphi\left(x_{2 i}\right)-\varphi\left(x_{2 i-2}\right)\right]+f(x)
\end{aligned}
$$

Here let's assign $A_{i}(x)=K\left(x, x_{2 i-1}^{*}\right)\left[\varphi\left(x_{2 i}\right)-\varphi\left(x_{2 i-2}\right)\right] \quad$ for $i=1,2,3,4$, then (10) becomes

$$
\bar{u}(x)=\sum_{i=1}^{4} A_{i}(x) u\left(x_{2 i-1}^{*}\right)+f(x)
$$

Then if the values $x_{1}^{*}, x_{3}^{*}, x_{5}^{*}, x_{7}^{*}$ are substituted into the equation (11), then the following system is obtained and solution is found by using Maple 18 as follows

$$
\begin{gathered}
\left(\begin{array}{cccc}
0.5945 & -0.1295 & -0.0892 & -0.0695 \\
-0.4081 & 0.8646 & -0.0961 & -0.0772 \\
-0.4132 & -0.1470 & 0.8902 & -0.0922 \\
-0.4209 & -0.1645 & -0.1303 & 0.8853
\end{array}\right) . \\
\left(\begin{array}{l}
u\left(x_{1}^{*}\right) \\
u\left(x_{3}^{*}\right) \\
u\left(x_{5}^{*}\right) \\
u\left(x_{7}^{*}\right)
\end{array}\right)=\left(\begin{array}{l}
0.0023 \\
0.1237 \\
0.2859 \\
0.4759
\end{array}\right) \Rightarrow\left(\begin{array}{l}
u\left(x_{1}^{*}\right) \\
u\left(x_{3}^{*}\right) \\
u\left(x_{5}^{*}\right) \\
u\left(x_{7}^{*}\right)
\end{array}\right)=\left(\begin{array}{l}
0.2853 \\
0.4207 \\
0.6102 \\
0.8413
\end{array}\right)
\end{gathered}
$$

Then, this solution is substituted back into (11) and simplified by Maple 18 to get

$$
\begin{aligned}
& \bar{u}(x)=0.282737088933138- \\
& 0.140452902234602 \cdot x^{2}-\frac{1}{6} \cdot x+x \sqrt{x}
\end{aligned}
$$

which is pretty close to the exact solution

$$
\begin{gathered}
u(x)=0.3058111302- \\
0.1289085929 \cdot x^{2}-\frac{1}{6} \cdot x+x \sqrt{x}
\end{gathered}
$$

As the number of subintervals " $n$ " increased, the accuracy in the approximate solution increases and the 
Table 1 Comparison determinant of the coefficient matrix in (12) and the approximate solution, as $\mathrm{n}$ increases.

\begin{tabular}{llll}
\hline$n$ & $\operatorname{det}(A)$ & $\bar{u}(x)$ & $u(x)$ \\
\hline 4 & 0.2658 & $0.2827-0.14045 \cdot x^{2}-\frac{1}{6} \cdot x+x \sqrt{x}$ & $0.3058-0.1289 \cdot x^{2}-\frac{1}{6} \cdot x+x \sqrt{x}$ \\
16 & 0.2625 & $0.3041-0.1300 \cdot x^{2}-\frac{1}{6} \cdot x+x \sqrt{x}$ & $0.3058-0.1289 \cdot x^{2}-\frac{1}{6} \cdot x+x \sqrt{x}$ \\
64 & 0.2622 & $0.3057-0.1290 \cdot x^{2}-\frac{1}{6} \cdot x+x \sqrt{x}$ & $0.3058-0.1289 \cdot x^{2}-\frac{1}{6} \cdot x+x \sqrt{x}$ \\
256 & 0.2622 & $0.3058-0.1289 \cdot x^{2}-\frac{1}{6} \cdot x+x \sqrt{x}$ & $0.3058-0.1289 \cdot x^{2}-\frac{1}{6} \cdot x+x \sqrt{x}$ \\
\hline
\end{tabular}

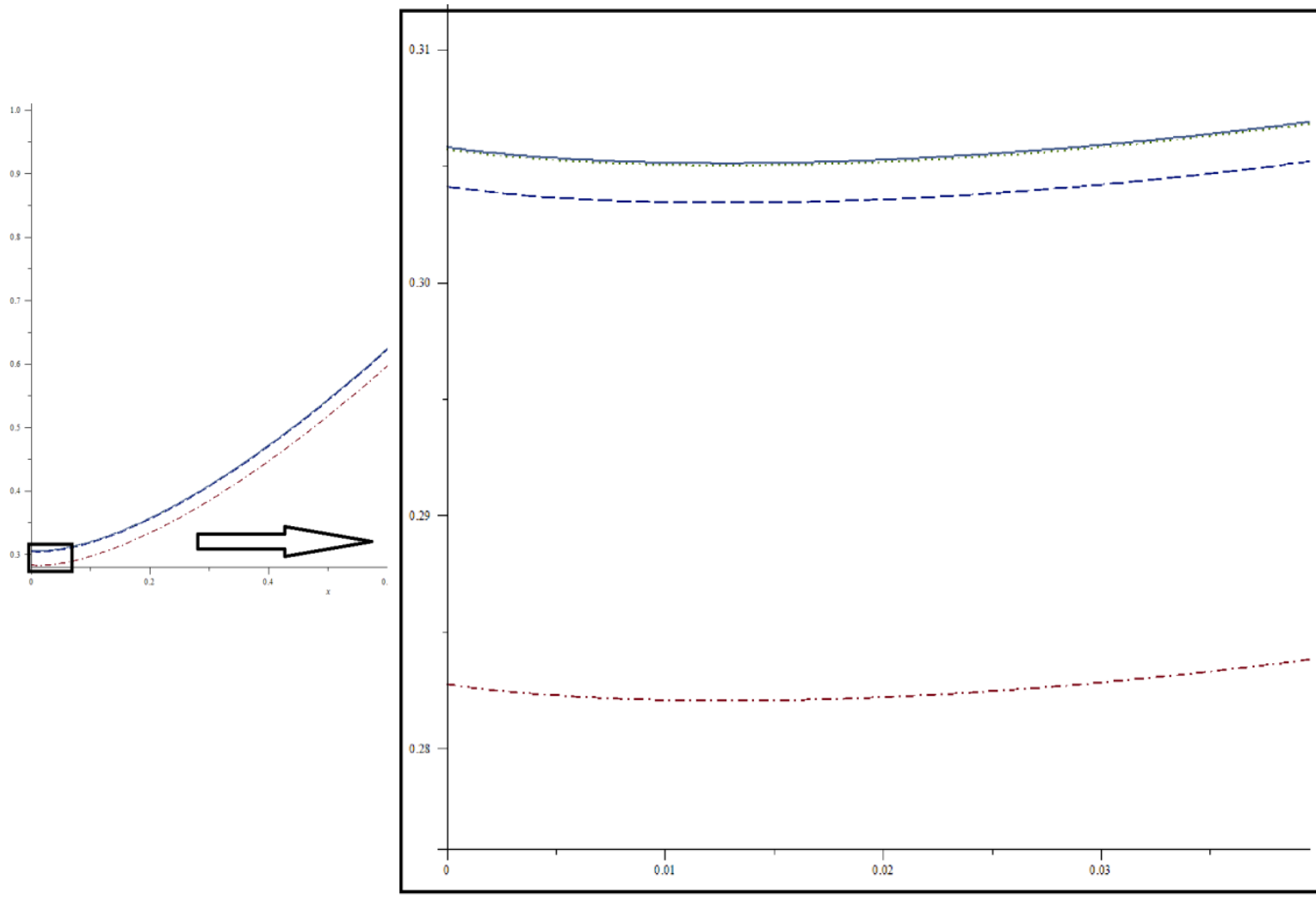

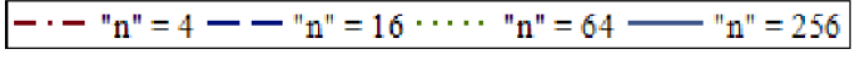

Fig. 1 Comparison graphs of the approximate solutions as $\mathrm{n}$ increases.

error decreases. The following table 1 shows how the approximate solution approaches the exact solution as the number of subintervals " $n$ " increases.

In the following figure 1, we have Maple 18 plot the solutions of the table 1 . It can be observed that as the number of subintervals " $n$ " increases, the graph of the solutions are accumulating around the exact solution which is close enough to the solution of $n=256$.

\section{Algorithm for solving Lfsiesk by Using the GMR in Maple 18}

The following screenshot is the algorithm of the approximate solution of the written by using Maple 18 . Any LFSIESK can be solved using this algorithm by changing its inputs in any accuracy. 


\section{Using the Generalized Midpoint Rule}

restart

with(LinearAlgebra) :

with(plots) :

\section{Inputs}

$K:=(x, s) \rightarrow 1+x^{2} \cdot s: f:=x \rightarrow-\frac{x^{2}}{4}-\frac{x}{6}+x \cdot \operatorname{sqrt}(x): p:=x \rightarrow \ln (1+\operatorname{sqrt}(x)): t:=x \rightarrow 0:$

$n:=4: a:=0.0: b:=1.0:$ lambda $:=1:$

\section{$\mathbf{U}(\mathbf{x})$}

Solver_General $:=\operatorname{proc}(K, p, t, f, a, b, 1$ ambda $:=1, n:=100)::$ function;

local invp := $x \rightarrow($ solve $(x=p(y), y)), h:=\frac{b-a}{2 \cdot n}, y, x::$ list, Id, $A, i, j, C, F, P r, u, U::$ list,

for $i$ from 0 by 2 to $2 \cdot n$ do $x_{i}:=a+i \cdot h$; end do; for $i$ from 1 to $n$ do $x_{2 \cdot i-1}:=i n v p\left(\frac{p\left(x_{2 \cdot i}\right)+p\left(x_{2 \cdot i-2}\right)}{2}\right)$; end do;

Id := IdentityMatrix $(n) ; A:=\operatorname{Matrix}(\mathrm{n})$;

for $\mathrm{i}$ from 1 to $\mathrm{n}$ dofor $\mathrm{j}$ from 1 ton do $A(i, j):=1$ ambda $\cdot K\left(x_{2 \cdot i-1}, x_{2 \cdot j-1}\right) \cdot\left(p\left(x_{2 \cdot j}\right)-p\left(x_{2 \cdot j-2}\right)\right)$; end do; end do;

$F:=\operatorname{Matrix}(\mathrm{n}, 1)$; for i from 1 ton $\operatorname{do} F(i, 1):=f\left(x_{2 \cdot i-1}\right)$; end do;

$\operatorname{Pr}:=\operatorname{Multiply}($ MatrixInverse $(I d-A), F)$; for $i$ from 1 to $n$ do $U_{i}:=\operatorname{Pr}(i, 1)$; end do;

$u:=y \rightarrow$ add $\left(\right.$ lambda $\left.\cdot K\left(y, x_{2 \cdot i-1}\right) \cdot\left(p\left(x_{2 \cdot i}\right)-p\left(x_{2 \cdot i-2}\right)\right) \cdot U[i], i=1 . . n\right)+f(y) ; u$

end proc:

\section{Conclusion}

In this work, the generalized midpoint rule is applied to solve LFSIESK. In a numerical example, the approximate results are compared with respect to the number of subintervals " $n$ " and plotted their graphs. It has been observed that, as the number of subintervals " $n$ " is increased, a very good accuracy can be obtained.

\section{References}

[1] Wolfgang H. (1995), Integral Equations; theory and numerical treatment, Birkhauser, Basel, Germany

[2] Delves L.M., Walsh J., (1974) Numerical Solution of Integral Equations, Clarendon, Oxford, England

[3] Atkinson K., Han W., (2009) Theoretical and Numerical Analysis, Springer, New York, USA

[4] Majeed S.M., (2014) Modified Midpoint Method for Solving System of Linear Fredholm Integral Equations of the Second Kind, American Journal of Applied
Mathematics, Volume 2. No 5, 155-161

[5] Фихтенгольц Г.М., (1996) Курс дифференциального и интегрального исчисления, Физматлит, Москва, Россия

[6] Asanov A., Muslu I., Yanik S., (2014) Approximation Solution of Linear Fredholm-Stieltjes Integral Equations of Second Kind Using Generalized Trapezoid Quadrature Approximation Methods, Исследования по Интегро-дифференциальным Уравнениям, Выпуск 46, 195-200

[7] Asanov A. , Chelik M.H., Abdujabbarov M. (2011) Approximating the Stieltjes Integral Using the Generalized Midpoint Rule, Matematika, Volume 27, Number 2, 139-148

[8] Gadjiev A.D., Aral A., (2007) The Estimates of Approximation by using a new type of weighted modulus of continuity, Computers and Mathematics with Applications, Volume 54, 127-135

[9] Anastassiou G.A., (1999) Inequalities for Local Moduli of Continuity, Applied Mathematics Letters, Volume 12, $7-12$ 